\title{
Prediction of optimal cardiac resynchronization by vectors extracted from electrograms in dyssynchronous canine hearts
}

Citation for published version (APA):

Engels, E. B., Strik, M., van Middendorp, L. B., Kuiper, M., Vernooy, K., \& Prinzen, F. W. (2017).

Prediction of optimal cardiac resynchronization by vectors extracted from electrograms in dyssynchronous canine hearts. Journal of Cardiovascular Electrophysiology, 28(8), 944-951.

https://doi.org/10.1111/jce.13241

Document status and date:

Published: 01/08/2017

DOI:

10.1111/jce. 13241

Document Version:

Publisher's PDF, also known as Version of record

\section{Document license:}

Taverne

Please check the document version of this publication:

- A submitted manuscript is the version of the article upon submission and before peer-review. There can be important differences between the submitted version and the official published version of record.

People interested in the research are advised to contact the author for the final version of the publication, or visit the DOI to the publisher's website.

- The final author version and the galley proof are versions of the publication after peer review.

- The final published version features the final layout of the paper including the volume, issue and page numbers.

Link to publication

\footnotetext{
General rights rights.

- You may freely distribute the URL identifying the publication in the public portal. please follow below link for the End User Agreement:

www.umlib.nl/taverne-license

Take down policy

If you believe that this document breaches copyright please contact us at:

repository@maastrichtuniversity.nl

providing details and we will investigate your claim.
}

Copyright and moral rights for the publications made accessible in the public portal are retained by the authors and/or other copyright owners and it is a condition of accessing publications that users recognise and abide by the legal requirements associated with these

- Users may download and print one copy of any publication from the public portal for the purpose of private study or research.

- You may not further distribute the material or use it for any profit-making activity or commercial gain

If the publication is distributed under the terms of Article $25 \mathrm{fa}$ of the Dutch Copyright Act, indicated by the "Taverne" license above, 


\title{
Prediction of optimal cardiac resynchronization by vectors extracted from electrograms in dyssynchronous canine hearts
}

\author{
Elien B. Engels PhD ${ }^{1}$ (D) | Marc Strik MD, PhD ${ }^{1,2}$ ｜ Lars B. van Middendorp MD, PhD ${ }^{1}$ | \\ Marion Kuiper BSc ${ }^{1}$ | Kevin Vernooy MD, $\mathrm{PhD}^{1,2}$ | Frits W. Prinzen $\mathrm{PhD}^{1}$
}

${ }^{1}$ Department of Physiology, Cardiovascular Research Institute Maastricht (CARIM), Maastricht University, Maastricht, the Netherlands

${ }^{2}$ Department of Cardiology, Maastricht University Medical Center, Maastricht, the Netherlands

\section{Correspondence}

Frits W. Prinzen, PhD, Department of Physiology, Maastricht University, P.O. Box 616, 6200MD, Maastricht, The Netherlands.

Email:frits.prinzen@maastrichtuniversity.nl

Funding Information

This work was supported within the framework of Center for Translational Molecular Medicine (www.ctmm.nl), Project COHFAR (Congestive Heart Failure and Arrhythmia) [Grant number 01C-203], and supported by the Dutch Heart Foundation.

F.W.P. has received research grants from Medtronic, St. Jude Medical, Sorin, MSD, and Biotronik. K.V. received research grants from Medtronic and St. Jude Medical. Other authors: No disclosures.

\begin{abstract}
Introduction: Proper optimization of atrioventricular (AV) and interventricular (VV) intervals can improve the response to cardiac resynchronization therapy (CRT). It has been demonstrated that the area of the QRS complex (QRSarea) extracted from the vectorcardiogram can be used as a predictor of optimal CRT-device settings. We explored the possibility of extracting vectors from the electrograms (EGMs) obtained from pacing electrodes and of using these EGM-based vectors (EGMVs) to individually optimize acute hemodynamic CRT response.

Methods and results: Biventricular pacing was performed in 13 dogs with left bundle branch block (LBBB) of which five also had myocardial infarction (MI), using 100 randomized AV-and VVsettings. Settings providing an acute increase in $\mathrm{LV} \mathrm{dP/dt}$ max $\geq 90 \%$ of the highest achieved value were defined as optimal. The prediction capability of QRSarea derived from the EGMV (EGMVQRSarea) was compared with that of QRS duration. EGMV-QRSarea strongly correlated to the change in LV dP/dt $t_{\max }(R=-0.73 \pm 0.19$ [LBBB] and $-0.66 \pm 0.14$ [LBBB + MI]), while QRS duration was more poorly related to $\mathrm{LV} d P / \mathrm{dt}_{\max }$ changes $(\mathrm{R}=-0.33 \pm 0.25$ [LBBB] and $-0.47 \pm$ 0.39 [LBBB + MI]). This resulted in a better prediction of optimal CRT-device settings by EGMVQRSarea than by QRS duration (LBBB: AUC $=0.89$ [0.86-0.93] vs. 0.76 [0.69-0.83], $\mathrm{P}<0.01$; $\mathrm{LBBB}+\mathrm{MI}: \mathrm{AUC}=0.91$ [0.84-0.99] vs. $0.82[0.59-1.00], \mathrm{P}=0.20$, respectively).
\end{abstract}

Conclusion: In canine hearts with chronic LBBB with or without MI, the EGMV-QRSarea predicts acute hemodynamic CRT response and identifies optimal $A V$ and $V V$ settings accurately. These data support the potency of EGM-based vectors as a noninvasive, easy and patient-tailored tool to optimize CRT-device settings.

\section{KEYWORDS}

cardiac resynchronization therapy, electrophysiology, electrogram, left bundle branch block, vector

\section{1 | INTRODUCTION}

Cardiac resynchronization therapy (CRT) has been shown to improve morbidity and mortality in patients with heart failure and left bundle branch block (LBBB). ${ }^{1}$ However, the response to CRT is heterogeneous, leaving a considerable amount of patients as nonresponders. ${ }^{2}$ One of the factors contributing to the nonresponse is a poor optimization of the CRT-device settings. ${ }^{3}$

The CRT-device settings that can be programmed are the time intervals between electrical stimulation of the right atrium and the ventricles (AV interval) and between the right (RV) and left ventricle (LV; VV interval). These settings determine LV filling characteristics and the degree of ventricular resynchronization. Currently, the techniques for in-hospital optimization of the CRT-device use echocardiographic ventricular filling parameters or LV systolic function variables. However, these methods are time consuming, have high measurement variability, and/or are invasive. ${ }^{4-6}$ While such a single optimization of the CRT-device settings is probably valuable, regular optimization, preferably in an automated fashion, seems more desirable. Therefore, new algorithms were developed using electrogram $(\mathrm{EGM})^{7,8}$ or electrocardiogram (ECG) ${ }^{9}$ signals. However, all these algorithms are based on parameters measured during intrinsic activation and estimate optimal stimulation based on average data from a group of patients. Therefore, in the current study we investigated the possibility to individually and automatically optimize the CRT-device settings continuously using signals acquired during the paced situation. 
An earlier study from our group in canine hearts with LBBB showed the importance of electrical resynchronization for hemodynamic response to CRT. ${ }^{10}$ Van Deursen et al. ${ }^{11,12}$ previously showed that a three-dimensional (3-D) vectorcardiogram (VCG) or two frontal plane vectors extracted from the ECG, reflect electrical interventricular dyssynchrony and are reliable and reproducible tools for CRTdevice optimization. It was also shown that the optimal AV and VV interval was predicted by the smallest QRS area. In the present study, we explored the possibility to derive vectors from the electrograms (EGMs) obtained from the pacing electrodes (EGM-based vector; EGMV) and to use the EGMV-based QRS area for CRT optimization in LBBB dog hearts with or without myocardial infarction (MI). This would allow automatic, individual, and virtually continuous $A V$ and $V V$ interval optimization.

\section{2 | METHODS}

Animal handling was performed according to the Dutch Law on Animal Experimentation and the European Directive for the Protection of Vertebrate Animals Used for Experimental and Other Scientific Purposes. The protocol was approved by the Animal Experimental Committee of Maastricht University.

\section{1 | Experimental model}

The experiments were performed in 13 mongrel dogs of either sex and unknown age, of whom eight had LBBB and five had LBBB in combination with MI. The protocol has been described previously. ${ }^{10}$ In short, anesthesia of the animals was induced by IV thiopental sodium administration and maintained by continuous infusion of midazolam ( $0.25 \mathrm{mg} / \mathrm{kg}$ per hour IV) and sufentanyl ( $3 \mu \mathrm{g} / \mathrm{kg}$ per hour IV). In the LBBB + MI group, transmural infarction was created by embolization of the LAD artery using a suspension of polyvinyl alcohol foam particles. After 4 weeks of MI (in LBBB + MI animals), or at the start of the protocol (LBBB animals), radiofrequency ablation was used to induce LBBB. The pacing protocol, as described in the next section, was performed 16 weeks after onset of LBBB, thus allowing for ventricular remodeling to occur. ${ }^{13} \mathrm{RV}$ and LV pressure catheters were positioned and the right atrial pacing lead was inserted transvenously. In addition, an octapolar electrode catheter was placed against the right side of the septum, from which the most distal electrode was used for RV apical pacing. ${ }^{10}$ After opening the chest, two multielectrode arrays holding 102 contact electrodes were placed around the heart to measure epicardial electrical potentials and a basal posterolateral electrode was selected for LV pacing (see Fig. 1A).
A

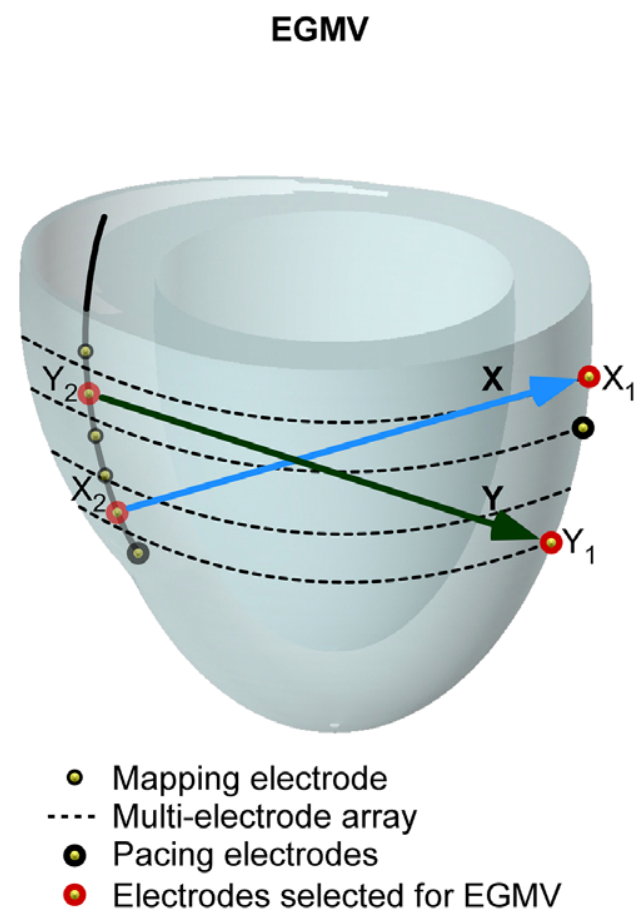

B

LBBB

LV pacing

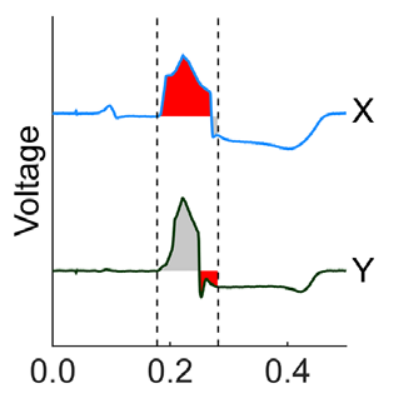

QRSarea

$=\left((3.09-0.20)^{2}+\right.$

$\left.(2.42-0.45)^{2}\right)^{1 / 2}$

$=3.50 \mathrm{Vs}$

\section{QRSarea \\ $=\left((0.61-2.89)^{2}+\right.$ \\ $\left.(0.70-1.80)^{2}\right)^{1 / 2}$ \\ $=2.54 \mathrm{Vs}$}

FIGURE 1 Flowchart of the EGMV derived QRSarea determination. Panel A indicates the position of the two epicardial multielectrode arrays and the multielectrode catheter in the RV. The electrodes indicated by a thick black circle are the pacing electrodes and the electrodes indicated by a thick red circle are the electrodes used to measure unipolar EGM signals. The bipolar " $X$ " vector is calculated by subtracting the EGM signal of the RV apical electrode $\left(X_{2}\right)$ from the LV basal electrode $\left(X_{1}\right)$ and the bipolar " $Y$ " vector is calculated by subtracting the signal of $Y_{2}$ from that of $Y_{1}$. The bipolar EGM vectors " $X$ " (blue) and " $Y$ " (green) are shown in panel B. The QRS complex is indicated by black dashed lines and the area under the curve is indicated in grey when positive and red when negative. The value of red areas is subtracted from that of the grey areas (panel C) [Color figure can be viewed at wileyonlinelibrary.com] 


\section{2 | Pacing protocol}

During the pacing protocol, the delay between the right atrium and either the RV (A-RV delay) or LV (A-LV delay) ranged from 50 to 230 milliseconds in steps of 20 milliseconds, resulting in a raster of 10 $\times 10$ and thus 100 different pacing settings. The occurrence of all 100 different combinations was randomly programmed. Every four steps of pacing settings were preceded by a baseline measurement that was equal to atrial pacing at a heart rate 10 BPM above intrinsic sinoatrial rate. These baseline measurements were used to calculate relative changes in $\mathrm{LV} \mathrm{dP} / \mathrm{dt}_{\max }$ measurements. Both hemodynamic and electrophysiological data were recorded for a minimum of two respiratory cycles.

\section{3 | Data analysis}

To capture the activation in the direction of the paced waves, four unipolar EGM signals were selected to form two bipolar EGMVs. The required EGMs were measured from two viable electrodes with the largest distance from each other on the octapolar electrode catheter (RV) and two LV electrodes, one just above and one just below the LV pacing electrode on the contact electrode bands. The " $X$ " vector was calculated by subtracting the signal of the apical RV EGM from the basal LV EGM, and the "Y" vector by subtracting the signal of the basal RV EGM from the apical LV EGM (Figs 1A, B). Using custom MATLAB R2010b (MathWorks, Natick, MA, USA) software, the QRS complex was detected using the Pan-Tompkins algorithm. ${ }^{14}$ Subsequently, the EGMV-derived area of the QRS complex (QRSarea; EGMVQRSarea) was calculated using the formula QRSarea $=\left(Q_{\text {RSarea }}{ }^{2}+\right.$ QRSarea $\left._{Y}{ }^{2}\right)^{1 / 2}$ where QRSarea ${ }_{X}$ and QRSarea are the area of the QRS complexes between the curve and baseline in vectors $X$ and $Y$, respectively (Fig. 1C).

The hemodynamic response was assessed by measuring the maximal rate of $L V$ pressure rise ( $L V d P / d t_{\text {max }}$ ). When a setting resulted in

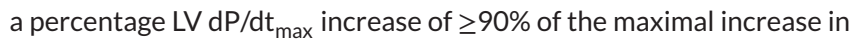

$\mathrm{LV} \mathrm{dP/dt} \mathrm{max}_{\max }$ compared to baseline (absolute surface peak), the setting was identified as optimal ( $\mathrm{CRT}_{\text {opt }}$ ).

For each setting, the variable was plotted in relation to the A-RV and A-LV delays in a 3-D surface plot. Quadratic fitting was applied to account for measurement variability.

\subsection{Statistical analysis}

Continuous data are presented as mean \pm standard deviation. Correlations between surface plots of percentage change in $\mathrm{LV} \mathrm{dP/dt}$ max compared to baseline and the surface plots of the electrophysiological variables EGMV-QRSarea and QRS duration were calculated using the Pearson correlation coefficient for each animal individually. Between all variables an average correlation coefficient and accompanying standard deviation was calculated combining all LBBB animals and all LBBB + MI animals. The classification performance of electrical variables in identifying the $\mathrm{CRT}_{\text {opt }}$ settings were evaluated by receiver operating characteristic (ROC) curve analysis. For each animal, a separate ROC curve was generated. From these individual ROC curves an average overall ROC curve was calculated using vertical averaging. Classification performances of QRS duration and EGMV-QRSarea were analyzed using the area under the ROC curve (AUC). The paired t-test was used to evaluate the significance of the difference in AUCs between the 2 variables, and the one-sample t-test was used to calculate their corresponding $95 \%$ confidence intervals. A P value $<0.05$ was considered statistically significant.

\section{3 | RESULTS}

The baseline and chronic characteristics of the eight LBBB and the five LBBB + MI animals are presented in Table 1. Creation of the LBBB increased QRS duration from 50 to 110 milliseconds. Between baseline and 16 weeks LBBB, LV systolic pressure and LV dP/dt $t_{\max }$ decreased while end-diastolic LV pressure and RV systolic pressure increased in both groups of animals.

TA B LE 1 Baseline electrocardiographic and hemodynamic characteristics of the eight LBBB animals and the five LBBB + MI animals, presented as mean \pm standard deviation

\begin{tabular}{|c|c|c|c|c|}
\hline \multirow[b]{2}{*}{ Variable } & \multicolumn{2}{|c|}{ LBBB Animals $(n=8)$} & \multicolumn{2}{|c|}{$\underline{\text { LBBB}+M I}$ Animals $(\mathrm{n}=5)$} \\
\hline & Baseline & Chronic & Baseline & Chronic \\
\hline Heart rate (BPM) & $89 \pm 15$ & $119 \pm 15$ & $101 \pm 1$ & $133 \pm 23$ \\
\hline PR interval (milliseconds) & $138 \pm 17$ & $162 \pm 34$ & $125 \pm 34$ & $131 \pm 12$ \\
\hline QRS duration (milliseconds) & $52 \pm 6$ & $108 \pm 8$ & $56 \pm 7$ & $108 \pm 17$ \\
\hline QTc interval (milliseconds) & $403 \pm 46$ & $398 \pm 21$ & $426 \pm 32$ & $406 \pm 25$ \\
\hline LV ESP (mmHg) & $94 \pm 8$ & $63 \pm 11$ & $98 \pm 13$ & $70 \pm 4$ \\
\hline LV EDP (mmHg) & $6 \pm 2$ & $9 \pm 3$ & $5 \pm 1$ & $7 \pm 5$ \\
\hline $\mathrm{LV} \mathrm{dP} / \mathrm{dt}_{\max }(\mathrm{mmHg} / \mathrm{s})$ & $1788 \pm 298$ & $1299 \pm 289$ & $1759 \pm 426$ & $1464 \pm 337$ \\
\hline $\mathrm{LV} \mathrm{dP} / \mathrm{dt}_{\min }(\mathrm{mmHg} / \mathrm{s})$ & $-2042 \pm 214$ & $-1240 \pm 130$ & $-2131 \pm 475$ & $-1561 \pm 190$ \\
\hline $\operatorname{RVESP}(\mathrm{mmHg})$ & $13 \pm 3$ & $23 \pm 9$ & $14 \pm 2$ & $27 \pm 12$ \\
\hline RV EDP (mmHg) & $3 \pm 3$ & $8 \pm 4$ & $3 \pm 1$ & $3 \pm 6$ \\
\hline $\mathrm{RV} \mathrm{dP} / \mathrm{dt}_{\max }(\mathrm{mmHg} / \mathrm{s})$ & $373 \pm 52$ & $507 \pm 73$ & $437 \pm 40$ & $677 \pm 200$ \\
\hline
\end{tabular}

$\mathrm{EDP}=$ end diastolic pressure; $\mathrm{ESP}=$ end systolic pressure. 


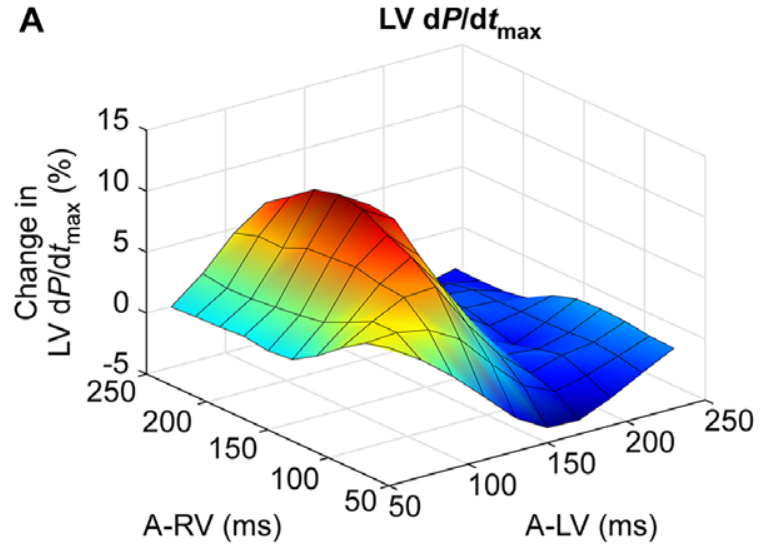

PR-interval $=141 \mathrm{~ms}$

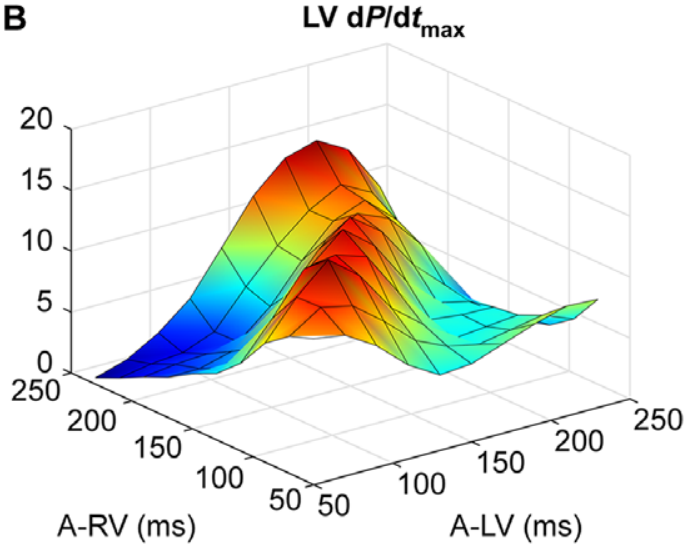

PR-interval $=201 \mathrm{~ms}$

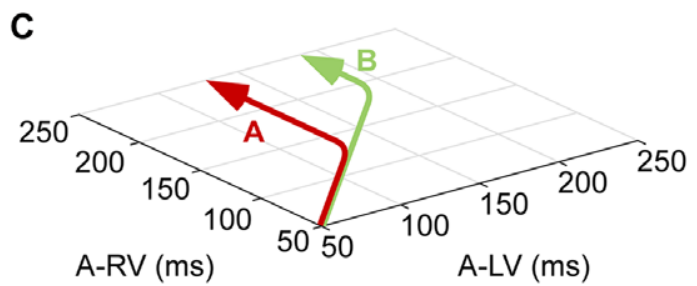

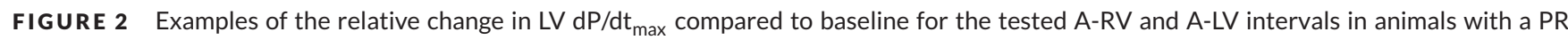
interval of 141 milliseconds (A) and 201 milliseconds (B). At the diagonal line in the horizontal plane $(y=x)$ the VV interval was 0 , points to the right of this line indicate RV pre-excitation and points to the left LV pre-excitation. A combination of a short A-LV and a long A-RV is equal to LV-only pacing, while a short A-RV and a long A-LV represents RV-only pacing. There are multiple combinations of A-RV and A-LV intervals resulting in a high change in $\mathrm{LV} \mathrm{dP/dt} \mathrm{max}_{\max }$ compared to baseline (the settings indicated by the red color). These settings form a "mountain ridge." The course of this ridge is indicated in Figure $C$. The red line indicates the ridge of animal $A$ and the green line of animal B. The leftward turn of the ridge of animal $A$ is earlier than the turn of animal $B$ [Color figure can be viewed at wileyonlinelibrary.com]

A

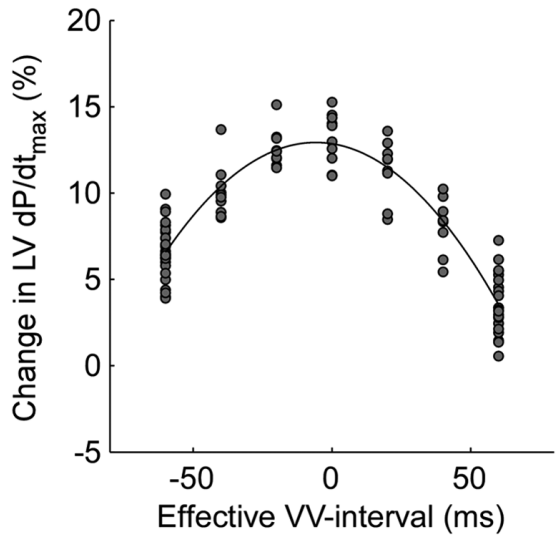

B

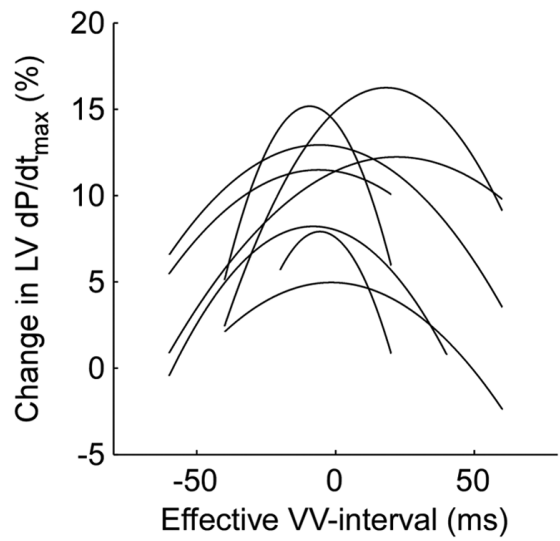

FIGURE 3 A: Typical example of the relationship between effective $\mathrm{VV}$ interval and improvement in maximum rate of LV pressure rise (LV $\mathrm{dP} / \mathrm{dt}_{\max }$ ) compared to baseline for all pacing configurations. B: Relationship between effective $\mathrm{VV}$ interval and change in LV $\mathrm{dP} / \mathrm{dt}_{\max }$ compared to baseline for all LBBB animals

\section{1 | Hemodynamic effect of different stimulation intervals during CRT}

Two examples of the hemodynamic changes at all 100 tested A-RV/ALV combinations are shown in Figure 2. LV- and RV-only pacing at short AV intervals (left and right corner in Fig. 2, respectively) caused only small changes in LV dP/dt $t_{\max }$ as compared to baseline. This was also the case during simultaneous biventricular (BiV) pacing at long $\mathrm{AV}$ intervals (top corner) because of loss of effective pacing. Several combinations of A-RV and A-LV intervals led to significant hemodynamic improvement. At short AV intervals, high $\mathrm{LV} \mathrm{dP/dt}$ max values were found during simultaneous BiV pacing, while at longer ARV intervals the optimum was found during LV pre-excitation, most likely related to fusion of LV pacing with intrinsic activation. ${ }^{10}$ These settings together form the "mountain ridge" of the surface plots, indicated by the red color (Figs 2A, B). This ridge was usually not straight, but showed a turn from the diagonal to the upward direction, 
A

PR-interval $=141 \mathrm{~ms}$ EGMV-QRSarea

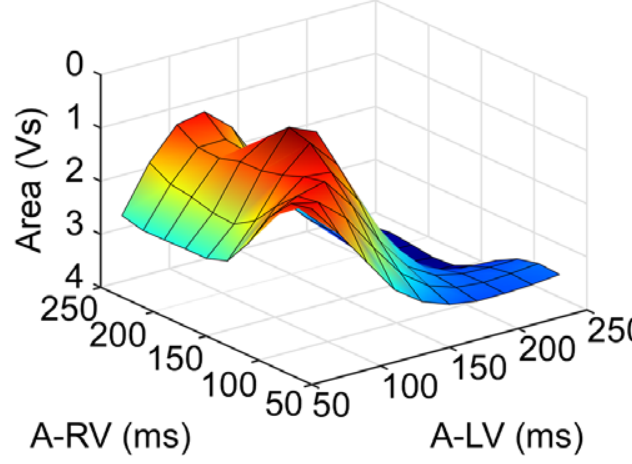

'B
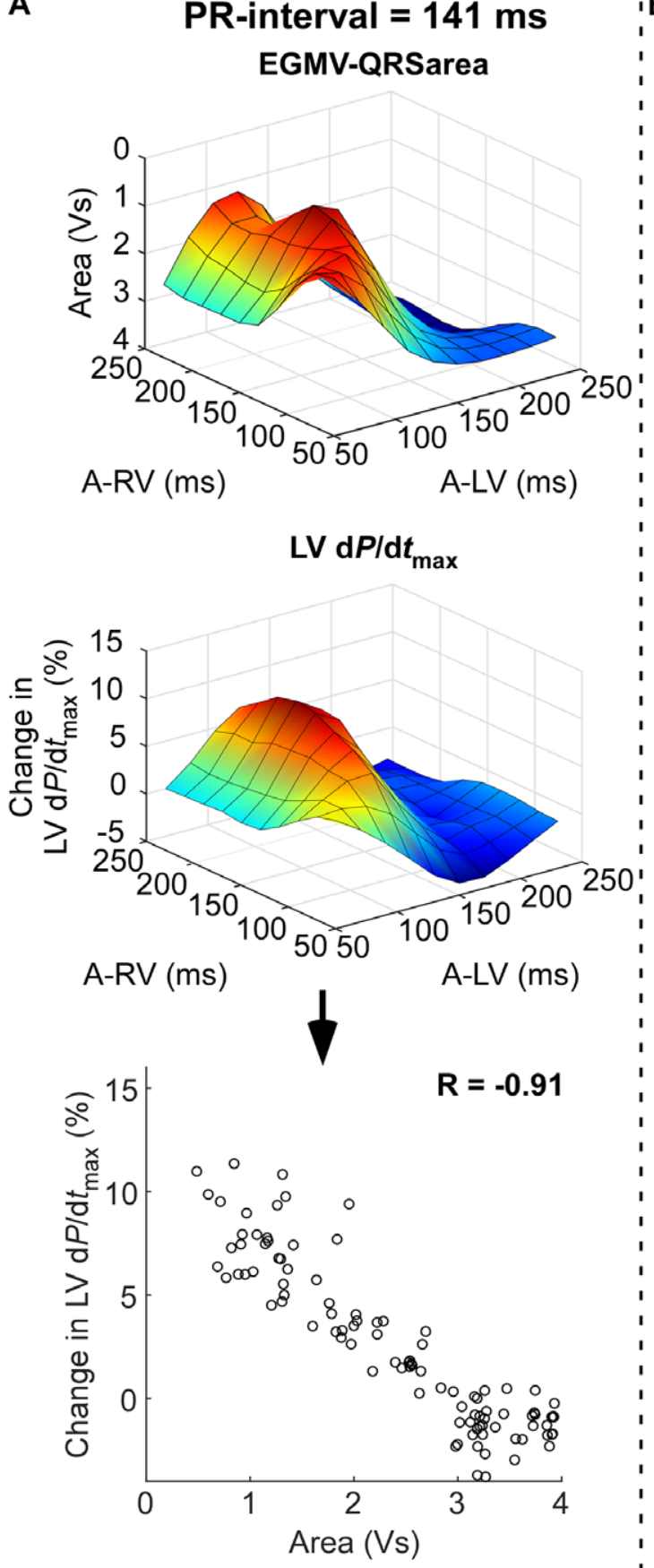

PR-interval $=201 \mathrm{~ms}$ EGMV-QRSarea

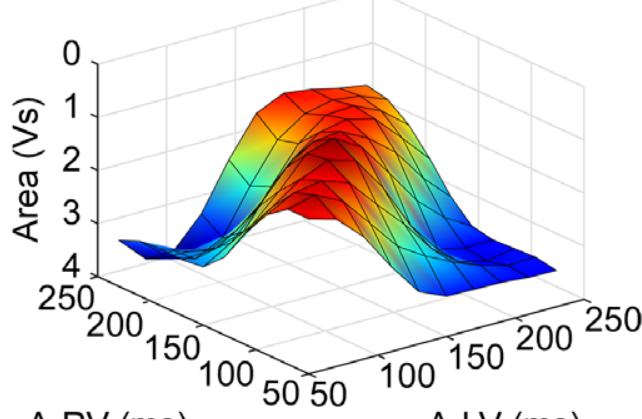

A-RV (ms)
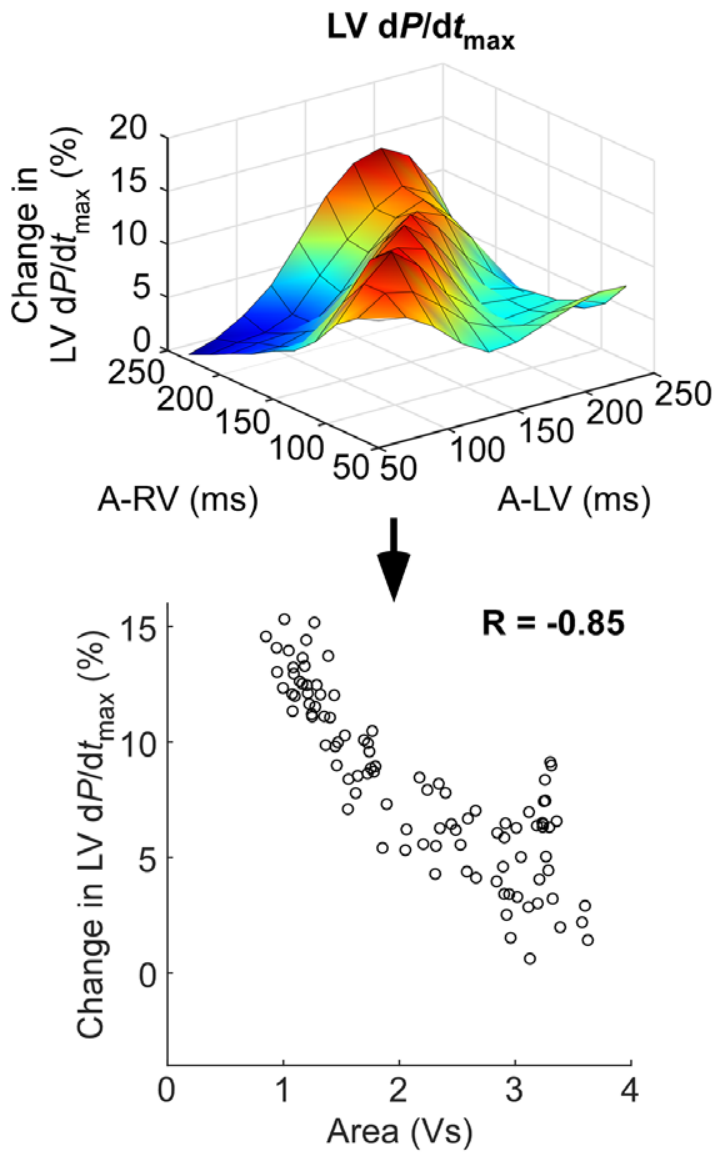

FIGURE 4 Examples of surface plots for QRSarea derived from the EGMV and the corresponding surface plots for the change in LV $\mathrm{dP} / \mathrm{dt} \mathrm{max}_{\text {ax }}$ compared to baseline for the same animals presented in Figure 2. For visual comparison between QRSarea and LV dP/dt $t_{\text {max }}$ surface plots, the QRS area axis was inverted. The agreement between the EGMV-QRSarea and LV dP/dt $\mathrm{max}_{\text {mar }}$ surface plots are indicated between the two surface plots. In the bottom figure, $\mathrm{LV} \mathrm{dP/dt}$ max is plotted against EGMV-QRSarea for all 100 different settings [Color figure can be viewed at wileyonlinelibrary.com]

indicating that highest $\mathrm{LV} \mathrm{dP} / \mathrm{dt}_{\max }$ values occurred during simultaneous BiV pacing at shorter AV-delays, but during LV pre-excitation at longer AV delays. In the two examples shown, this bending of the ridge occurred at a longer AV-delay in the animal with a longer intrinsic PR interval (Fig. 2C). When for each LBBB animal the effective $V V$ interval (the actual time delay between onset of activation of the RV apex and LV lateral wall ${ }^{15}$ ) was calculated, all points transferred to a single parabola (Fig. 3), indicating that at longer AV-delays the optimal hemodynamic response occurred during LV fusion pacing.

\subsection{EGMV prediction performance}

Figures $4 A$ and $B$ show the surface plots for QRSarea as derived from EGMV and the corresponding LV dP/dt $t_{\max }$ surface plot during all 100 tested A-RV/A-LV combinations of two LBBB animals with different intrinsic PR intervals. There was a good match between values of EGMV-QRSarea and LV dP/dt $t_{\max }$ with low values of EGMV-QRSarea matching large increases in LV $\mathrm{dP} / \mathrm{dt}_{\max }$. LV-only pacing or RV-only pacing resulted in high EGMV-QRSarea values and low $\mathrm{LV} \mathrm{dP} / \mathrm{dt}_{\max }$ 


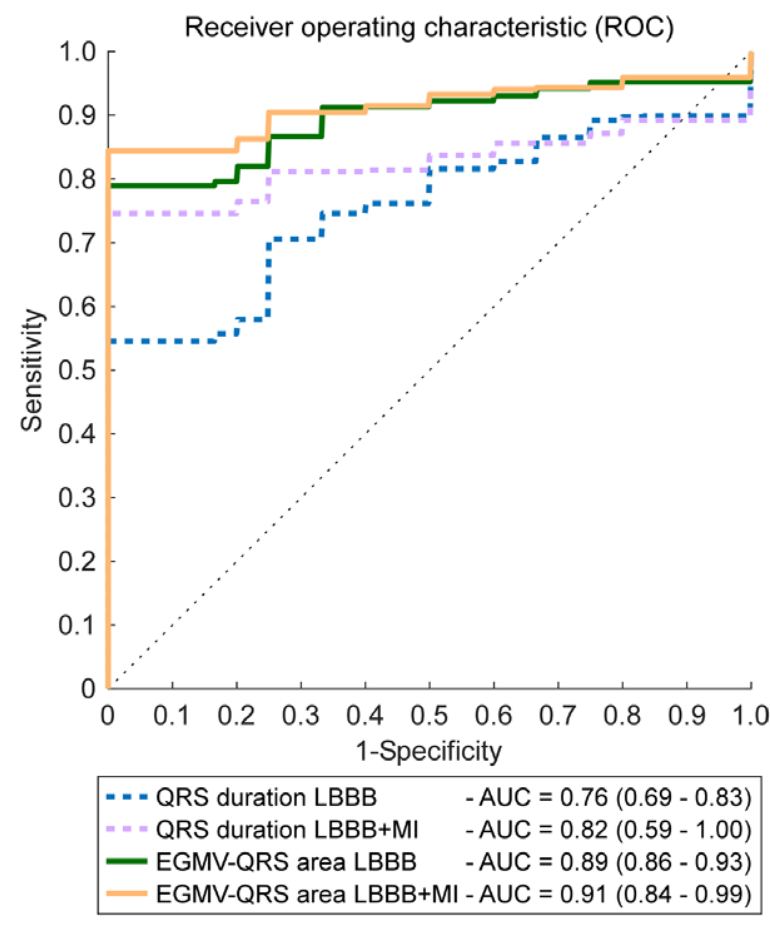

FIGURE 5 Receiver operating characteristics (ROC) curves for QRS duration (dashed blue [LBBB] and purple [LBBB + MI] lines) and EGMV-QRSarea (green [LBBB] and orange [LBBB + MI] line) for clas-

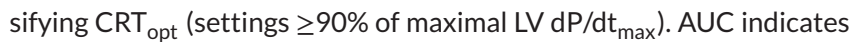
the ROC area under the curve which signifies the performance of each variable in identifying the $\mathrm{CRT}_{\text {opt }}$ settings [Color figure can be viewed at wileyonlinelibrary.com]

increases, while settings ranging from simultaneous BiV-pacing at short AV intervals to pronounced LV pre-excitation at longer A-RV intervals resulted in lower EGMV-QRSarea and higher $\mathrm{LV} \mathrm{dP} / \mathrm{dt}_{\max }$ increases. Combining all LBBB animals, there was a correlation of $-0.73 \pm 0.19$ between EGMV-QRSarea and LV dP/dt $t_{\max }$.

In the animal with a longer PR interval, a larger number of settings

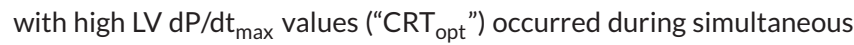
BiV pacing (before the leftward turn, Fig. 4B), whereas in the animal with a short PR interval many $\mathrm{CRT}_{\text {opt }}$ points occurred during fusion of intrinsic conduction with the paced activation wavefronts (after the turn, Fig. 4A). This was also reflected in the EGMV-QRSarea.

Similar results were found for the LBBB $+\mathrm{MI}$ animals. Settings with

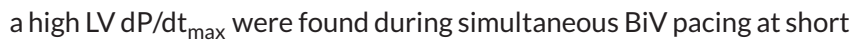
AV-delay and in settings of LV fusion pacing at longer A-RV delays. The A-RV delay at which this shift happened was again dependent on the PR interval. The correlation between EGMV-QRSarea and LV dP/dt $\max$ was $-0.66 \pm 0.14$ for all $\mathrm{LBBB}+\mathrm{Ml}$ animals.

\section{3 | ROC curves}

Figure 5 presents ROC curves that show the average classifying capabilities of EGMV-QRSarea and QRS duration to identify CRT opt $_{\text {. The }}$ AUC for the EGMV-QRSarea was high for both the LBBB (0.89 [0.860.93]) and LBBB + MI animals (0.91 [0.84-0.99]), while the QRS duration was less accurate in identifying the settings resulting in optimal hemodynamic improvement (LBBB: 0.76 [0.69-0.83], $\mathrm{P}<0.01$ compared to EGMV-QRSarea; LBBB + MI: 0.82 [0.59-1.00], P = 0.20 compared to EGMV-QRSarea). Indeed, the variation in QRS duration between different settings was quite small, making it difficult to select the best settings using QRS duration (Fig. 6). Furthermore, the A-RV and A-LV intervals resulting in a minimal QRS duration do not match the intervals resulting in a maximal increase in $\mathrm{LV} \mathrm{dP} / \mathrm{dt}_{\max }$ compared to baseline. This resulted in a low correlation between QRS duration and $\mathrm{LV} \mathrm{dP} / \mathrm{dt}_{\max }$ for both the LBBB $(\mathrm{R}=-0.33 \pm 0.25)$ and LBBB $+\mathrm{MI}$ animals $(R=-0.47 \pm 0.39)$.

\section{4 | DISCUSSION}

The present study performed in LBBB canine hearts with and without previous $\mathrm{MI}$ demonstrates that during an extensive pacing protocol with a wide range of $A V$ and $V V$ intervals, QRSarea derived from intracardiac electrodes can be used to optimize the acute hemodynamic response of CRT. This observation provides evidence that QRSarea derived from the electrograms obtained from intracardiac pacing electrodes can become an easy, noninvasive, accurate, and reproducible tool for individual optimization of $\mathrm{AV}$ and $\mathrm{VV}$ intervals in CRT.

\subsection{Individual optimization of CRT using vectors}

Previous studies from our group already demonstrated that during LVonly pacing in canines and patients, vectorcardiographic indices can be used for optimizing the AV interval. ${ }^{11,12}$ Furthermore, during BiV pacing, vectorcardiography could find the optimal VV interval. The current canine study shows that also the EGMV-QRSarea can be used for optimization of the $A V$ and $V V$ interval in CRT.

Several device manufacturers already use EGM signals to automatically optimize AV and VV interval settings. ${ }^{7-9}$ However, all of these methods are based on parameters measured during intrinsic activation and based on an estimation of optimal stimulation derived from average data from a group of patients. Doing so, they do not consider individual differences during intrinsic conduction or during pacing. None of these methods have shown superiority in CRT outcome compared to the use of standard (out of the box) delays or to a single echocardiographic optimization. ${ }^{16-18}$ Our concept of EGMV uses the signals during the paced situation to find the individual and optimal pace settings of the CRT-device. This might result in a better and more accurate CRT optimization, potentially leading to a better outcome after CRT in the long run. In order to illustrate this, combing all animals, LBBB and $\mathrm{LBBB}+\mathrm{MI}$, the observed optimal CRT response was compared to that at a setting where A-RV is set to $80 \%$ of the delay between atrial pace spike and the beginning of QRS complex and A-LV 20 milliseconds shorter (so a VV interval of -20 milliseconds). In the latter setting the increase in $\mathrm{LV} \mathrm{dP/dt} \mathrm{max}_{\max }$ was $11.4 \pm 3.9 \%$ which is significantly lower than using the proposed optimization technique $(16.2 \pm 4.0 \%$, $\mathrm{P}<0.01$ ).

For all animals combined the EGMV based QRSarea outperformed the paced $Q R S$ duration $(P<0.01)$. The $A V$ and $V V$ intervals corresponding to the shortest QRS duration do not correspond to the $\mathrm{AV}$ and $\mathrm{VV}$ interval resulting in a maximal increase in $\mathrm{LV} \mathrm{dP} / \mathrm{dt}_{\text {max }}$. While QRS duration is a frequently used method for CRT-device 


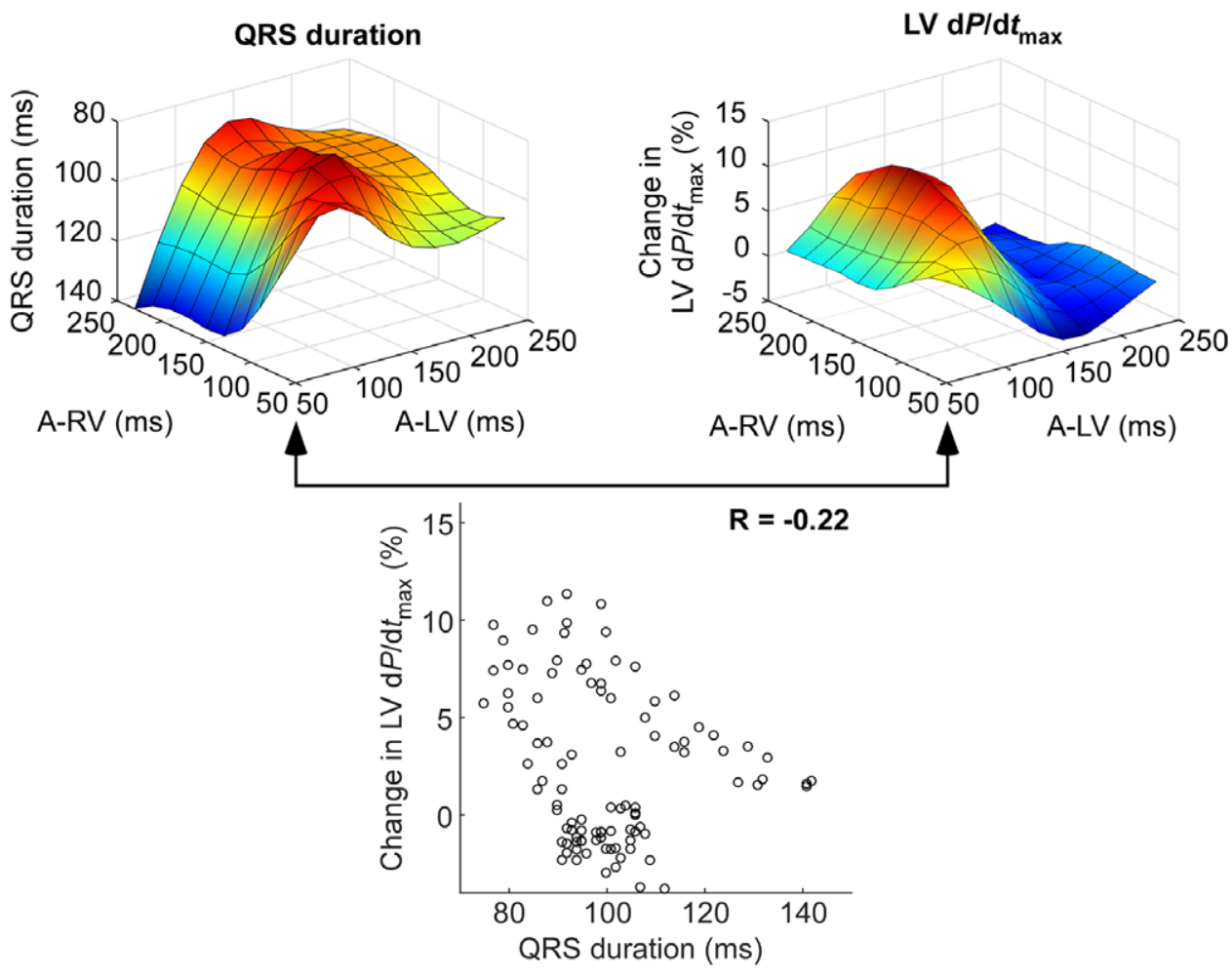

FIGURE 6 Relation between QRS duration and LV dP/dt $\mathrm{max}_{\text {max }}$ for the canine with a PR interval of 141 milliseconds. For visual comparison, the QRS duration-axis was inverted. In the bottom figure, $\mathrm{LV} \mathrm{dP/dt}$ max is plotted against QRS duration for all 100 different settings [Color figure can be viewed at wileyonlinelibrary.com]

optimization, several studies have also provided conflicting results about the role of QRS shortening as a predictor of CRT response. ${ }^{19-21}$ An explanation for these findings might be that QRS duration reflects the total ventricular activation time regardless of activation sequence, while QRSarea reflects opposing electrical forces, an indication of dyssynchronous electrical wavefronts. ${ }^{11,22}$ Of note, QRS duration cannot readily be determined from EGM signals, therefore, this would also not be feasible when used in an automated optimization as envisioned using our EGMV approach.

\section{2 | Potential clinical implications}

Extrapolation of experimental data to the clinical situation should always be done with care. However, it has already been shown that the QRSarea can be used to predict the best AV interval during LVonly pacing and the best $\mathrm{V} V$ interval during BiV pacing. ${ }^{12}$ Therefore, the results of this animal study might well be applicable in the clinical situation.

While in the present study, the EGMVs were calculated from four unpaced electrodes, a minimum of three is required. With the currently available pacing leads, one could use the two unpaced electrodes on a quadripolar LV lead and the RV ring of the RV lead. When using this approach in the LBBB experiments the AUC for EGMV-QRSarea was 0.84 (0.79-0.89) as compared to 0.89 (0.86-0.93) when using four electrodes. Therefore, our approach is also expected to be valid when using currently available pacing leads. Clearly, this technique should be validated in a patient population.
Implementing the relatively simple software to derive the EGMVQRSarea from these EGMs into the CRT-device would enable to automatically and frequently calculate EGMV-QRSarea and let the device find the $A V$ and $V V$ interval corresponding to a minimal EGMVQRSarea using an automated stepwise optimization algorithm. This provides a noninvasive and easy method for continuous optimization of the $\mathrm{AV}$ and $\mathrm{VV}$ intervals, also during different activity levels.

\section{3 | Limitations}

The current study only took the acute response to CRT into account using hemodynamic measurements. The acute CRT response may not correspond to CRT response on the long term. ${ }^{23}$ Moreover, while the canines had long-term LBBB introducing mild cardiac dysfunction, ${ }^{24-26}$ the quantitative response in patients with a more severe degree of heart failure might be different. However, Strik et al. ${ }^{27}$ showed that CRT effects were similar in canine hearts with LBBB alone or in combination with tachypacing induced heart failure, albeit that higher relative changes were seen in the heart failure animals. The present study shows comparable results in animals with LBBB only and in combination with MI, indicating that the algorithm is robust.

\section{5 | CONCLUSION}

In LBBB canine hearts with or without MI, QRSarea derived from two vectors extracted from two RV and two LV intracardiac EGMs 
predicts acute hemodynamic response and identifies optimal CRTdevice settings accurately. These data support the potency of a devicebased EGMV as a noninvasive and easy tool to individually and potentially continuously optimize the AV and VV intervals in CRT.

\section{REFERENCES}

1. Cleland J, Daubert JC, Erdmann E, et al. The effect of cardiac resynchronization on morbidity and mortality in heart failure. $N$ Engl J Med 2005;352:1539-1549.

2. European Heart Rhythm Association, European Society of Cardiology, Heart Rhythm Society, Heart Failure Society of America, American Society of Echocardiography, American Heart Association, European Association of Echocardiography, Heart Failure Association, Daubert JC, Saxon L, Adamson PB, et al. 2012 EHRA/HRS expert consensus statement on cardiac resynchronization therapy in heart failure: implant and follow-up recommendations and management. Heart Rhythm. 2012;9:1524-1576.

3. Mullens W, Grimm RA, Verga T, et al. Insights from a cardiac resynchronization optimization clinic as part of a heart failure disease management program. J Am Coll Cardiol. 2009;53:765-773.

4. Raphael CE, Kyriacou A, Jones S, et al. Multinational evaluation of the interpretability of the iterative method of optimisation of $A V$ delay for CRT. Int J Cardiol2013;168:407-413.

5. Auricchio A, Stellbrink C, Block M, et al. Effect of pacing chamber and atrioventricular delay on acute systolic function of paced patients with congestive heart failure. The Pacing Therapies for Congestive Heart Failure Study Group. The Guidant Congestive Heart Failure Research Group. Circulation 1999;99:2993-3001.

6. Pabari PA, Willson K, Stegemann B, et al. When is an optimization not an optimization? Evaluation of clinical implications of information content (signal-to-noise ratio) in optimization of cardiac resynchronization therapy, and how to measure and maximize it. Heart Fail Rev 2011;16:277-290.

7. Baker JH, 2nd, McKenzie J, 3rd, Beau S, et al. Acute evaluation of programmer-guided $\mathrm{AV} / \mathrm{PV}$ and $\mathrm{VV}$ delay optimization comparing an IEGM method and echocardiogram for cardiac resynchronization therapy in heart failure patients and dual-chamber ICD implants. J Cardiovasc Electrophysiol 2007;18:185-191.

8. Krum H, Lemke B, Birnie D, et al. A novel algorithm for individualized cardiac resynchronization therapy: rationale and design of the adaptive cardiac resynchronization therapy trial. Am Heart $J$ 2012;163:747-752 e1.

9. Gold MR, Niazi I, Giudici M, et al. A prospective comparison of AV delay programming methods for hemodynamic optimization during cardiac resynchronization therapy. J Cardiovasc Electrophysiol 2007;18:490496.

10. Strik M, van Middendorp LB, Houthuizen P, et al. Interplay of electrical wavefronts as determinant of the response to cardiac resynchronization therapy in dyssynchronous canine hearts. Circ Arrhythm Electrophysiol 2013;6:924-931.

11. van Deursen CJ, Strik M, Rademakers LM, et al. Vectorcardiography as a tool for easy optimization of cardiac resynchronization therapy in canine left bundle branch block hearts. Circ Arrhythm Electrophysiol 2012;5:544-552.

12. van Deursen CJ, Wecke L, van Everdingen WM, et al. Vectorcardiography for optimization of stimulation intervals in cardiac resynchronization therapy. J Cardiovasc Transl Res 2015;8:128-137.

13. van Middendorp LB, Kuiper M, Munts C, et al. Local microRNA-133a downregulation is associated with hypertrophy in the dyssynchronous heart. ESC Heart Failure. 2017; https://doi.org/10.1002/ehf2.12154
14. Pan J, Tompkins WJ. A real-time QRS detection algorithm. IEEE Trans Biomed Eng 1985;32:230-236.

15. Vernooy K, Verbeek XA, Cornelussen RN, et al. Calculation of effective $\mathrm{VV}$ interval facilitates optimization of $\mathrm{AV}$ delay and $\mathrm{V} V$ interval in cardiac resynchronization therapy. Heart Rhythm 2007;4:75-82.

16. Ellenbogen KA, Gold MR, Meyer TE, et al. Primary results from the SmartDelay determined AV optimization: a comparison to other AV delay methods used in cardiac resynchronization therapy (SMART-AV) trial: A randomized trial comparing empirical, echocardiography-guided, and algorithmic atrioventricular delay programming in cardiac resynchronization therapy. Circulation 2010;122: 2660-2668.

17. Wang D, Yu H, Yun T, et al. Long-term clinical effects of programmerguided atrioventricular and interventricular delay optimization: intracardiac electrography versus echocardiography for cardiac resynchronization therapy in patients with heart failure. $J$ Int Med Res 2013;41:115-122.

18. Martin DO, Lemke B, Birnie D, et al.; Adaptive CRT Study Investigators. Investigation of a novel algorithm for synchronized left-ventricular pacing and ambulatory optimization of cardiac resynchronization therapy: results of the adaptive CRT trial. Heart Rhythm. 2012;9:18071814.

19. Perego GB, Chianca R, Facchini M, et al. Simultaneous vs. sequential biventricular pacing in dilated cardiomyopathy: an acute hemodynamic study. Eur J Heart Fail 2003;5:305-313.

20. Nawar A, El-Hoseiny R, Ragab D, Al-Aziz AA. V-V delay interval optimization in CRT using echocardiography compared to QRS width in surface ECG. Egyptian Heart J 2012;64:127-133.

21. Vidal B, Tamborero D, Mont L, et al. Electrocardiographic optimization of interventricular delay in cardiac resynchronization therapy: a simple method to optimize the device. J Cardiovasc Electrophysiol 2007;18:1252-1257.

22. Varma N, Jia P, Rudy Y. Electrocardiographic imaging of patients with heart failure with left bundle branch block and response to cardiac resynchronization therapy. J Electrocardiol 2007;40:S174-8.

23. Bogaard MD, Houthuizen $P$, Bracke FA, et al. Baseline left ventricular $\mathrm{dP} / \mathrm{dtmax}$ rather than the acute improvement in $\mathrm{dP} / \mathrm{dtmax}$ predicts clinical outcome in patients with cardiac resynchronization therapy. Eur J Heart Fail 2011;13:1126-1132.

24. Vernooy K, Verbeek XA, Peschar M, et al. Left bundle branch block induces ventricular remodelling and functional septal hypoperfusion. Eur Heart J 2005;26:91-98.

25. Vernooy K, Cornelussen RN, Verbeek XA, et al. Cardiac resynchronization therapy cures dyssynchronopathy in canine left bundle-branch block hearts. Eur Heart J 2007;28:2148-2155.

26. Kerckhoffs RC, Lumens J, Vernooy K, et al. Cardiac resynchronization: insight from experimental and computational models. Prog Biophys Mol Biol. 2008;97:543-561.

27. Strik M, van Deursen CJ, van Middendorp LB, et al. Transseptal conduction as an important determinant for cardiac resynchronization therapy, as revealed by extensive electrical mapping in the dyssynchronous canine heart. Circ Arrhythm Electrophysiol. 2013;6: 682-689.

How to cite this article: Engels EB, Strik M, van Middendorp LB, Kuiper M, Vernooy K, Prinzen FW. Prediction of optimal cardiac resynchronization by vectors extracted from electrograms in dyssynchronous canine hearts. J Cardiovasc Electrophysiol. 2017;28:944-951. https://doi.org/10.1111/jce.13241 